\title{
Low value of whole-body dual-modality [18fffluorodeoxyglucose positron emission tomography/computed tomography in primary staging of stage I-II nasopharyngeal carcinoma: a nest case-control study
}

\author{
Bei-Bei Xiao ${ }^{1,2} \cdot$ Qiu-Yan Chen ${ }^{1,2} \cdot$ Xue-Song Sun ${ }^{1,2} \cdot \mathrm{Ji}^{-B i n} \mathrm{Li}^{1,3} \cdot$ Dong-hua Luo ${ }^{1,2} \cdot$ Rui Sun $^{1,2} \cdot$ Da-Feng Lin ${ }^{1,2}$. \\ Xu Zhang ${ }^{1,4}$. Wei Fan ${ }^{1,4}$. Xiao-Fei Lv ${ }^{1,5}$. Lu-Jun Han ${ }^{1,5}$. Yue-Feng Wen ${ }^{1,2} \cdot$ Li Yuan $^{1}$ • Shan-Shan Guo ${ }^{1,2}$. \\ Li-Ting Liu $^{1,2}$. Sai-Lan Liu ${ }^{1,2}$ - Qing-Nan Tang ${ }^{1,2}$ (1) Y Yu-Jing Liang ${ }^{1,2} \cdot$ Xiao-Yun Li ${ }^{1,2}$. Chao Lin ${ }^{1,2}$ - Ling Guo ${ }^{1,2}$. \\ Hai-Qiang Mai ${ }^{1,2} \cdot$ Lin-Quan Tang ${ }^{1,2}$
}

Received: 21 May 2020 / Revised: 9 October 2020 / Accepted: 5 November 2020 / Published online: 8 January 2021

(C) The Author(s) 2021

\begin{abstract}
Objectives The value of using PET/CT for staging of stage I-II NPC remains unclear. Hence, we aimed to investigate the survival benefit of PET/CT for staging of early-stage NPC before radical therapy.

Methods A total of 1003 patients with pathologically confirmed NPC of stages I-II were consecutively enrolled. Among them, 218 patients underwent both PET/CT and conventional workup ([CWU], head-and-neck MRI, chest radiograph, liver ultrasound, bone scintigraphy) before treatment. The remaining 785 patients only underwent CWU. The standard of truth (SOT) for lymph node metastasis was defined by the change of size according to follow-up MRI. The diagnostic efficacies were compared in 218 patients who underwent both PET/CT and CWU. After covariate adjustment using propensity scoring, a cohort of 872 patients (218 with and 654 without pre-treatment PET/CT) was included. The primary outcome was overall survival based on intention to treat.

Results Retropharyngeal lymph nodes were metastatic based on follow-up MRI in 79 cases. PET/CT was significantly less sensitive than MRI in detecting retropharyngeal lymph node lesions $(72.2 \%$ [62.3-82.1] vs. 91.1\% [84.8-97.4], $p=$ 0.004). Neck lymph nodes were metastatic in 89 cases and PET/CT was more sensitive than MRI (96.6\% [92.8-100.0] vs. $76.4 \%$ [67.6-85.2], $p<0.001)$. In the survival analyses, there was no association between pre-treatment PET/CT use and improved overall survival, progression-free survival, local relapse-free survival, regional relapse-free survival, and distant metastasis-free survival.
\end{abstract}

Bei-Bei Xiao and Qiu-Yan Chen contributed equally to this work and share the first authorship.

The senior authors Hai-Qiang Mai and Lin-Quan Tang contributed equally to this work.

Hai-Qiang Mai

Maihq@sysucc.org.cn

Lin-Quan Tang

tanglq@sysucc.org.cn

1 Sun Yat-sen University Cancer Center, State Key Laboratory of Oncology in South China, Collaborative Innovation Center for Cancer Medicine, Guangdong Key Laboratory of Nasopharyngeal Carcinoma Diagnosis and Therapy, 651 Dongfeng Road East, Guangzhou 510060, People's Republic of China

2 Department of Nasopharyngeal Carcinoma, Sun Yat-sen University Cancer Center, 651 Dongfeng Road East, Guangzhou 510060, People's Republic of China
3 Department of Clinical Research, Sun Yat-sen University Cancer Center, 651 Dongfeng Road East, Guangzhou 510060, People's Republic of China

4 Department of Nuclear Medicine, Sun Yat-sen University Cancer Center, 651 Dongfeng Road East, Guangzhou 510060, People's Republic of China

5 Department of Medical Imaging, Sun Yat-sen University Cancer Center, 651 Dongfeng Road East, Guangzhou 510060, People's Republic of China 
Conclusions This study showed PET/CT is of little value for staging of stage I-II NPC patients at initial imaging.

Key Points

- PET/CT was more sensitive than MRI in detecting neck lymph node lesions whereas it was significantly less sensitive than MRI in detecting retropharyngeal lymph node lesions.

- No association existed between pre-treatment PET/CT use and improved survival in stage I-II NPC patients.

Keywords Nasopharyngeal carcinoma $\cdot$ PET/CT $\cdot$ MRI $\cdot$ Neoplasm staging

$\begin{array}{ll}\text { Abbreviations } \\ \text { AJCC } & \text { American Joint Committee on Cancer } \\ \text { CCRT } & \text { Concurrent chemoradiotherapy } \\ \text { CLN } & \text { Cervical lymph node } \\ \text { CR } & \text { Complete response } \\ \text { CWU } & \text { Conventional workup } \\ \text { DMFS } & \text { Distant metastasis-free survival } \\ \text { EBV } & \text { Epstein-Barr virus } \\ \text { ECOG } & \text { Eastern Cooperative Oncology Group } \\ \text { ICT } & \text { Induction chemotherapy } \\ \text { IQR } & \text { Interquartile range } \\ \text { LN } & \text { Lymph node } \\ \text { LRFS } & \text { Local relapse-free survival } \\ \text { LRRFS } & \text { Locoregional relapse-free survival } \\ \text { MRI } & \text { Magnetic resonance imaging } \\ \text { NCCN } & \text { National Comprehensive Cancer Network } \\ \text { NPC } & \text { Nasopharyngeal carcinoma } \\ \text { NPV } & \text { Negative predictive value } \\ \text { PD } & \text { Progressive disease } \\ \text { PFS } & \text { Progression-free survival } \\ \text { PPV } & \text { Positive predictive value } \\ \text { PR } & \text { Partial response } \\ \text { PSM } & \text { Propensity score matching } \\ \text { RLN } & \text { Retropharyngeal lymph node } \\ \text { RRFS } & \text { Regional recurrence-free survival } \\ \text { RT } & \text { Radiotherapy } \\ \text { SD } & \text { Stable disease } \\ \text { SOT } & \text { The standard of truth } \\ & \end{array}$

\section{Introduction}

Nasopharyngeal carcinoma (NPC) is a malignant tumor with a worldwide incidence of $0.5-1.0 / 100,000$ per year, but occurs with a much higher incidence rate in southern China and Southeast Asia, in which it is endemic with an incidence rate of 30/100,000 persons per year [1,2]. The examinations for the initial staging of NPC comprise magnetic resonance imaging (MRI) of the head and neck, chest radiograph, liver ultrasound, bone scintigraphy (conventional workup [CWU]), and/or [18F]fluorodeoxyglucose positron emission tomography/computed tomography (PET/CT). The value of PET/CT and CWU at the initial staging of NPC has been extensively studied. Previous studies have reported about the superiority of MRI in detecting local tumor involvement and RLN (retropharyngeal lymph node) metastasis while PET/CT is advantageous in demonstrating CLN (cervical lymph node) metastasis and occult distant metastasis [3-5]. Besides, Lin et al demonstrated that PET/CT was useful in revealing occult distant metastases among NPC patients with advanced node disease [5-7]. Moreover, our previous study demonstrated that the NPC patients with stage N2-3 and Epstein-Barr virus (EBV) DNA $\geq 4000$ copies $/ \mathrm{mL}$ would benefit more from $\mathrm{PET} / \mathrm{CT}$ to detect distant metastasis [8]. With regard to local recurrence and residual NPC, the PET/CT was superior to MRI in diagnostic value based on the results of a metaanalysis [9]. Thus, it can be concluded that PET/CT is very important in the primary staging of NPC patients.

However, in relation to early-stage (stage I-II) NPC, there were few studies concerning the clinical benefit of PET/CT in this specific population. Besides, most studies focusing on the accuracy of PET/CT and CWU in NPC lacked long-term follow-up [3]. Furthermore, the 2019 guidelines of National Comprehensive Cancer Network (NCCN) recommended that PET/CT should be considered for NPC patients to detect distant metastases [10], evidence on clinical benefit of PET/CT in early-stage NPC is still lacking and worth studying. Therefore, we conducted this longitudinal cohort study to investigate the clinical benefit of PET/CT in early-stage NPC patients.

\section{Materials and methods}

\section{Patients}

From January 2007 to December 2014, 1003 consecutive patients histologically diagnosed with stage I-II NPC based on CWU in Sun Yat-sen University Cancer Center were enrolled in this study. Among them, 218 patients underwent both PET/ CT and CWU before treatment and the remaining 795 patients only underwent $\mathrm{CWU}$. The eligibility criteria were as follows: (1) histologically diagnosed WHO type II or III NPC; (2) clinical stage I or II (American Joint Committee on Cancer [AJCC] 8th staging system) based on CWU; (3) no history of a second malignant tumor; and (4) Eastern Cooperative Oncology Group (ECOG) performance score $\leq 2$. All examinations were performed within 2 weeks, and all patients were restaged based on the 8th AJCC staging manual. The study 
was approved by the clinical research ethics committee of Sun Yat-sen University Cancer Center and informed consent was obtained from all participants.

Considering potential confounders, propensity score matching (PSM) was performed among 1003 patients grouped by the examination method (PET/CT + CWU or CWU alone), and a well-balanced cohort including 218 patients (PET/CT plus CWU) and 654 patients (CWU only) was identified.

\section{PET/CT and MRI imaging}

PET/CT and MRI imaging information were included in the supplemental material.

\section{Image interpretation}

Head-and-neck MRI imaging was interpreted by two experienced radiologists. Regarding the neck and retropharyngeal lymph nodes on MRI, a diagnosis of metastatic lymph node was made if the presence of necrosis or extracapsular spread or cluster lymph nodes $(\geq 3)$ was detected. As for the size of lymph node, if the shortest axial diameter of the retropharyngeal node was $5 \mathrm{~mm}$ or greater, the lymph node was considered positive [11]. Similarly, the 10 -mm shortest axial diameter was the borderline size of positive CLN [12]. Two experienced nuclear medicine physicians interpreted PET/CT images without knowing patients' MRI results and clinical information [7, 13]. Lymph nodes were identified as positive if they showed significantly different tracer uptake compared to the background [14]. Any discrepancy was discussed by the research team and reached a consensus. The PET/CT results were added to CWU findings to make the final decision-making. Whether PET/CT results modified patients' clinical classification and subsequent treatment strategies were recorded.

\section{The standard of truth}

For patients in which PET/CT detected suspicious metastatic lesions, the validation was determined by assessing the histopathology or subsequent imaging examinations. The suspicious metastatic lesion was considered positive only if positive pathology result was obtained or at least two imaging examinations have positive results or suspicious lesions have progression within 1-year follow-up.

All patients were checked every 3 months within 12 months after initial diagnosis [4, 13]. According to the MRI imaging performed at 3 months after the entire treatment, a retropharyngeal or cervical lymph node lesion was determined as true positive if it was evaluated as showing partial response (PR), complete response (CR), or progressive disease (PD) based on the Response Evaluation Criteria in Solid Tumors

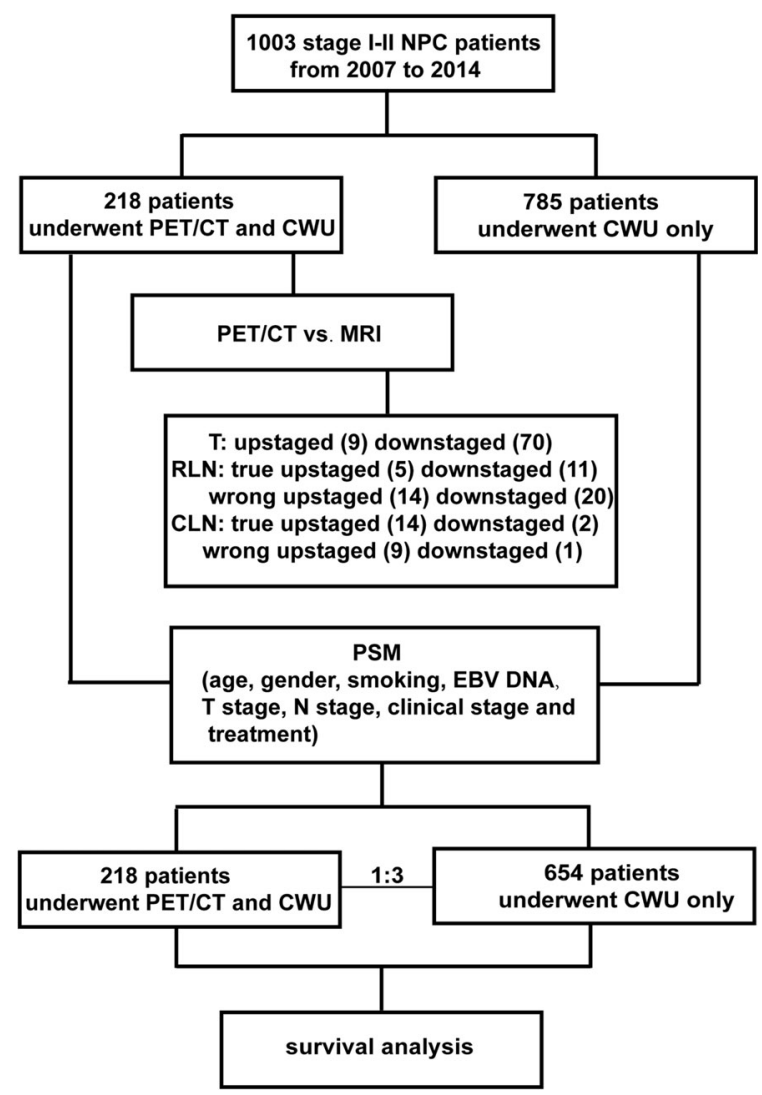

Fig. 1 Flowchart of this study

[15]. If the lymph node showed stable disease (SD) at 3 months after treatment, regular follow-up examinations for 1 year were compulsory. If the lymph node was found to enlarge by $120 \%$ or more in size within 1 year of follow-up, it was considered a true-positive lymph node, otherwise it was identified as true negative [16]. Distant metastasis detected within 1 year of follow-up was defined as proof of primary metastasis and false-negative screening.

\section{Treatment and follow-up}

All eligible patients were treated with intensity-modulated radiation therapy (IMRT). Target volumes were delineated slice-by-slice on treatment planning CT scans by using an individualized delineation protocol. Generally, a cumulative dose of $66 \sim 72$ Gy was administered for the planning target volume of the primary gross tumor volume (GTVnx) and the GTV of the involved lymph nodes (GTVnd), with 1.8-2.1 Gy per fraction and five daily fractions per week. All potential metastatic CLN drainage areas were prophylactically irradiated with at least $50 \mathrm{~Gy}$. Among 1003 patients, 426 patients were assigned to radiotherapy (RT) only, 378 patients received concurrent chemoradiotherapy (CCRT), 82 patients underwent the combination of induction chemotherapy 
Fig. 2 T- and N-staging discrepancies between PET/CT and head-and-neck MRI. $(\mathbf{a}, \mathbf{b})$ Female, 59 years old, T2N0M0 NPC. PET/CT image (left). Contrast-enhanced T1-weighted MRI image (right). PET/CT did not detect the parapharyngeal lesion that was found by MRI (red arrow). (c, d) Male, 57 years old, T2N1M0 NPC. PET/CT image (left). Contrast-enhanced T1weighted MRI image (right). PET/CT distinguished positive neck lymph node (red arrow) while it was regarded as a morphological benign lymph node in MRI
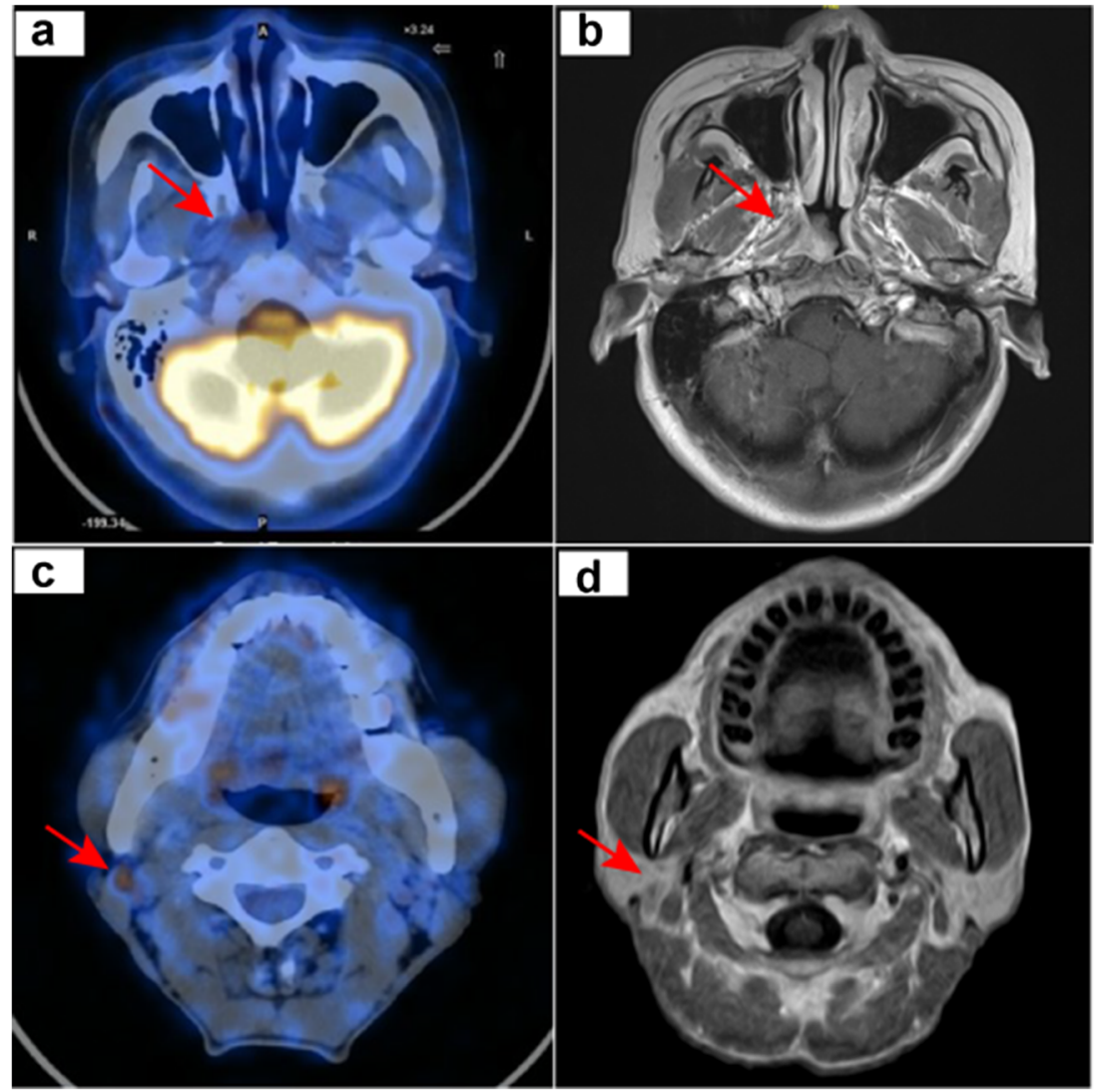

(ICT) and RT, and 117 patients received ICT plus CCRT. Among 218 patients who underwent PET/CT, 90 patients were assigned to radiotherapy (RT) alone, 78 patients received concurrent chemoradiotherapy (CCRT), 19 patients underwent induction chemotherapy (ICT) plus RT, and 31 patients received ICT and CCRT.

After the completion of treatment, follow-up examinations were performed every 3 months in the first 2 years, every 6 months in the following 3 years, and once a year thereafter [10]. Comprehensive physical examinations including nasopharyngoscopy, serum EBV DNA measurement, MRI of the head and neck, chest radiography, abdominal sonography, and bone scan were performed at each follow-up visit. If the tumor relapsed or distant metastasis was suspected, biopsy or targeted imaging methods were used to differentiate benign from malignant lesions.

\section{Statistical analysis}

PSM and covariate adjustment were performed using appropriate $\mathrm{R}$ software package. The independent variables thought to possibly affect study outcomes included age, gender, smoking, EBV DNA level, T category, $\mathrm{N}$ category, clinical stage, and treatment modality. The dependent variable was whether PET/CT had been performed. Finally, PSM with a matching ratio of 3 identified a cohort of 872 patients.
Survival rates were compared using Kaplan-Meier analysis with log-rank tests. Overall survival (OS), i.e., the time from diagnosis to the date of death from any reason or the date of the last follow-up, was the primary study outcome [17]. Progression-free survival (PFS) was the time from diagnosis to cancer progression or death from any reason. Local relapse-free survival (LRFS) or regional recurrence-free survival (RRFS) was calculated as the interval from diagnosis to local relapse or death from any reason, regional recurrence or death from any reason, respectively, while locoregional relapse-free survival (LRRFS) was calculated as the time from diagnosis to locoregional reoccurrence or death from any reason. Distant metastasis-free survival (DMFS) referred to the time from diagnosis to the date of distant metastasis or death from any reason. Patients lost to follow-up or alive without distant metastasis or locoregional recurrence at the last followup visit had their data censored. We estimated and compared the 5-year survival rates of PET/CT + CWU and CWU groups. Multivariable Cox regression models with an enter method were built to identify dependent prognostic factors of early-stage NPC using subsets of data after PSM.

McNemar's paired-sample test or $\chi^{2}$ test was applied to determine whether the sensitivity, specificity, positive predictive value, and negative predictive value of PET/CT and MRI in diagnosing lymph node metastasis were significantly different in the PET/CT + CWU cohort. 
Statistical analyses were performed with R software package (Version 3.5.2, http://www.r-project.org/) and SPSS 25 (IBM). All statistical tests were two-sided, and $p$ values of $<$ 0.05 were considered significant.

\section{Results \\ Staging discrepancies between PET/CT and CWU}

In the PET/CT + CWU group, 133 and 85 patients were respectively diagnosed as category $\mathrm{T} 1$ and $\mathrm{T} 2$ based on MRI (Fig. 1). PET/CT altered the T category diagnosis in 79 patients, with nine patients being upstaged and 70 downstaged due to the discrepancy in determining parapharyngeal space involvement (Fig. 2a, b). As for $\mathrm{N}$ category, 101 and 117 patients were classified as N0 and $\mathrm{N} 1$, respectively. PET/CT upstaged 28 patients and downstaged 24 patients.

A total of 218 patients primarily underwent both PET/CT and CWU and showed no distant metastasis. PET/CT staging did not change patients' major treatment strategy. Although PET/CT modified the planned target volume and dose in $25(11.5 \%)$ patients since their $\mathrm{N}$ categories were truly changed according to the standard of truth (SOT), with 15 upstaged and 10 downstaged, the treatment plans of 193 (88.5\%) patients were not changed. Among those 193 patients, both imaging tests achieved consistent results in 105 (54.4\%) patients and PET/CT wrongly upstaged or downstaged MRI-based $\mathrm{N}$ category in 27 patients according to the SOT. Besides, PET/CT upstaged and downstaged MRI-based T category in 9 and 70 patients, respectively, without knowing if it was changed truly because of lack for SOT. Given that PET/CT was inferior to MRI in detecting soft tissue lesions, therefore, the $\mathrm{T}$ staging changed by PET/CT did not obviously influence patients' treatment strategies.

\section{Retropharyngeal lymph node metastasis}

According to the SOT, RLNs were identified as metastatic in 79 cases, of which 57 were detected by PET/CT and 72 by MRI. PET/CT was significantly less sensitive than MRI in detecting RLN lesions (72.2\% [62.3-82.1] vs. 91.1\% [84.897.4], $p=0.004$ ) (Figure S1a). The specificity, positive predictive value (PPV), and negative predictive value (NPV) of PET/CT and MRI for detecting RLN lesions were $88.5 \%$ vs. $90.6 \%, 78.1 \%$ vs. $84.7 \%$, and $84.8 \%$ vs. $94.7 \%$, respectively (Table 1). Fifty (22.9\%) patients presented with inconsistent results between PET/CT and MRI. MRI correctly diagnosed 31 cases out of 50 patients. PET/CT missed truly metastatic RLNs in 20 patients and showed false-positive results in 14 patients (Fig. 3). Among the 16 patients with truly metastatic 
Fig. 3 Detecting discrepancies of retropharyngeal and neck lymph nodes between PET/CT and headand-neck MRI. (a, b) Male, 45 years old, T2N1M0 NPC. PET/ $\mathrm{CT}$ image (left). Contrastenhanced T1-weighted MRI image (right). Metastatic retropharyngeal lymph node was detected by MRI (red arrow) but not PET/CT. (c, d) Male, 50 years old, T2N1M0 NPC. PET/CT image (left). Contrast-enhanced T1weighted MRI image (right). Metastatic retropharyngeal lymph node was obvious on MRI (red arrow), while it disappeared on PET/CT because of the shadow of the primary lesion
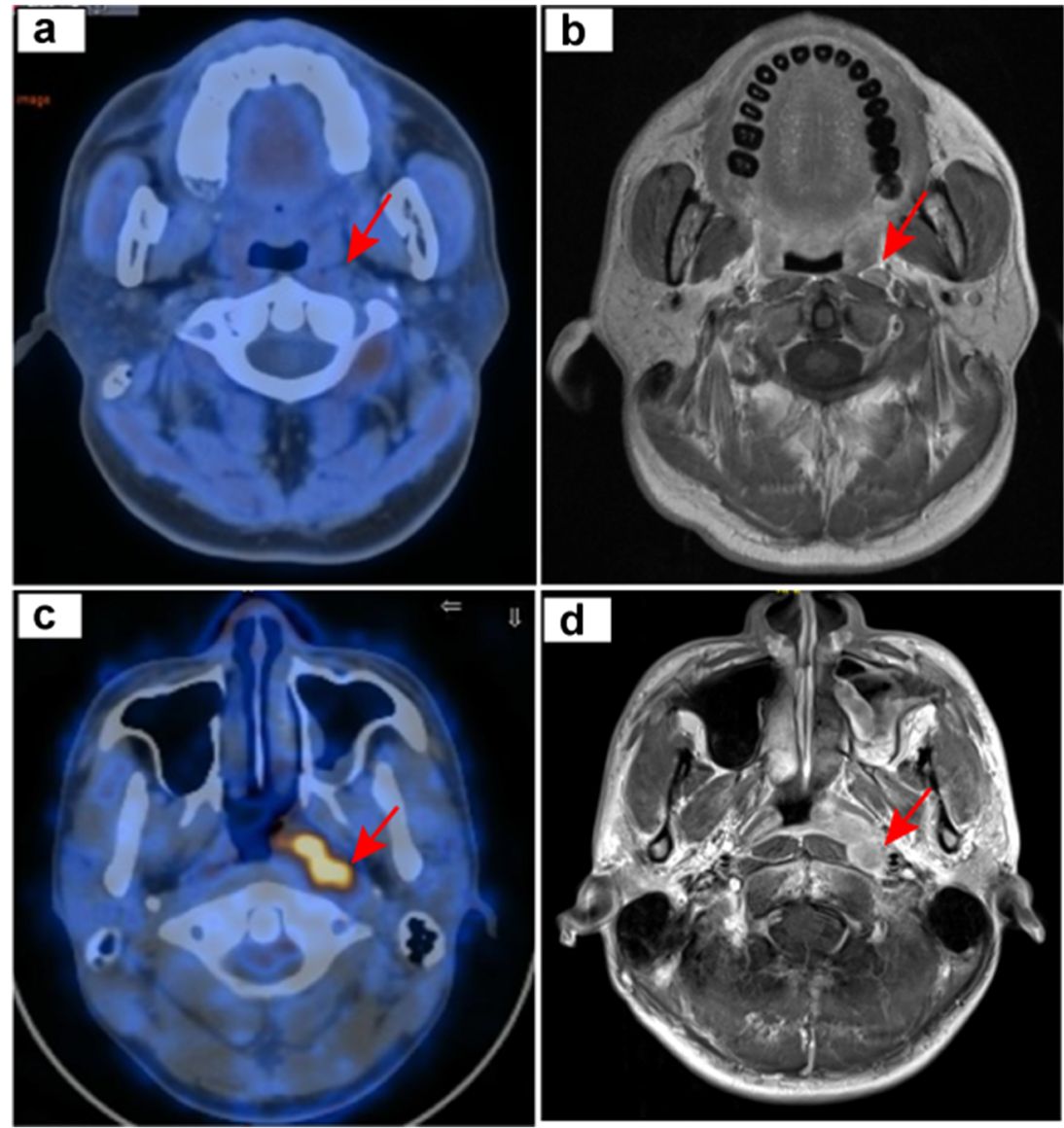

LNs who were diagnosed correctly by PET/CT, five had small RLNs with a shortest axial diameter less than $5 \mathrm{~mm}$ but with high FDG uptake. All doubtful LNs were treated with radical radiotherapy without the acquirement of retropharyngeal nodal biopsy due to the anatomical inaccessibility.

\section{Cervical lymph node and distant metastasis}

CLNs at initial diagnosis in 89 patients were confirmed to be metastatic based on SOT. Among them, 86 patients and 68 patients were correctly diagnosed by PET/CT and MRI accordingly. PET/CT was more sensitive than MRI (96.6\% [92.8-100.0] vs. 76.4\% [67.6-85.2], $p<0.001$ ) (Figure $\mathrm{S} 1 \mathrm{~b})$. The comparisons of specificity, PPV, and NPV between PET/CT and MRI for detecting CLNs were $72.9 \%$ vs. $96.1 \%, 71.1 \%$ vs. $93.2 \%$, and $96.9 \%$ vs. $85.5 \%$, respectively (Table 1). Among 26 (11.9\%) patients showing inconsistent CLN results, PET/ CT presented true-positive findings in 14 patients with morphologically benign LNs in MRI (Fig. 2c, d) and true-negative findings in two patients with morphologically malignant lymph nodes on MRI. Nonetheless, PET/CT yielded wrong results in ten patients, which included false-positive results in nine patients and false-negative result in one patient. In terms of distant metastasis, all 218 patients underwent both $\mathrm{PET} / \mathrm{CT}$ and CWU before initial treatment and no distant metastasis was detected. However, one patient developed liver metastasis within 1 year after diagnosis.

\section{Survival analysis in the PET/CT and CWU groups}

The PSM cohort included 218 NPC patients who underwent both PET/CT and CWU and 654 patients who only underwent CWU. No imbalanced covariate existed between these two groups. The demographics and clinical characteristics of patients were shown in Table 2. The median follow-up period in the PET/CT (PETCT + CWU) group was 63.0 (interquartile range [IQR], 55.7-74.2) months. During the follow-up, four $(1.8 \%)$ patients died, of which three $(1.4 \%)$ died of disease progression and one $(0.5 \%)$ died from other reasons. Nine $(4.1 \%)$ patients showed disease progression, of which 7 (3.2\%) developed local occurrence, 3 (1.4\%) patients developed regional occurrence, and $5(2.3 \%)$ showed distant metastasis. In the CWU group, the median follow-up period was 67.3 months (IQR, 55.6-83.3 months). Twenty-six (4.0\%) patients died, of which 22 
Table 2 Baseline characteristics of observational dataset and PSM dataset $(n=872)$

\begin{tabular}{|c|c|c|c|c|c|c|}
\hline \multirow[t]{2}{*}{ Characteristic } & \multicolumn{3}{|c|}{ Observational dataset $(n=1003)$} & \multicolumn{3}{|c|}{ PSM dataset $(n=872)$} \\
\hline & $\begin{array}{l}\mathrm{PET} / \mathrm{CT}+\mathrm{CWU} \\
(n=218)\end{array}$ & $\begin{array}{l}\text { CWU } \\
(n=785)\end{array}$ & $p$ & $\begin{array}{l}\mathrm{PET} / \mathrm{CT}+\mathrm{CWU} \\
(n=218)\end{array}$ & $\begin{array}{l}\text { CWU } \\
(n=654)\end{array}$ & $p$ \\
\hline Age (yr) & & & 0.167 & & & 0.334 \\
\hline Median(range) & $44.5(35-51)$ & $47(20-81)$ & & $44.5(35-51)$ & $46(39-54)$ & \\
\hline$\leq 45$ & $119(54.6)$ & $387(49.3)$ & & $119(54.6)$ & $342(52.3)$ & \\
\hline$>45$ & $99(45.4)$ & $398(50.7)$ & & $99(45.4)$ & $312(47.7)$ & \\
\hline Gender & & & 0.083 & & & 0.743 \\
\hline Female & $47(21.6)$ & $215(27.4)$ & & $47(21.6)$ & $148(22.6)$ & \\
\hline Male & $171(78.4)$ & $570(72.6)$ & & $171(78.4)$ & $506(77.4)$ & \\
\hline T stage & & & 0.020 & & & 0.810 \\
\hline $\mathrm{T} 1$ & $133(61.0)$ & $409(52.1)$ & & $133(61.0)$ & $393(60.1)$ & \\
\hline $\mathrm{T} 2$ & $85(39.0)$ & $376(47.9)$ & & $85(39.0)$ & $261(39.9)$ & \\
\hline $\mathrm{N}$ stage & & & 0.992 & & & 0.906 \\
\hline No & $101(46.3)$ & $364(46.4)$ & & $101(46.3)$ & $306(46.8)$ & \\
\hline N1 & $117(53.7)$ & $421(53.6)$ & & $117(53.7)$ & $348(53.2)$ & \\
\hline Clinical stage & & & 0.175 & & & 1.000 \\
\hline I & 74 (33.9) & $229(29.2)$ & & 37 (17.6) & $125(19.8)$ & \\
\hline II & $144(66.1)$ & $556(70.8)$ & & 173 (82.4) & $505(80.2)$ & \\
\hline EBV DNA & & & 0.012 & & & 0.139 \\
\hline$<4000$ & $168(77.1)$ & $662(84.3)$ & & $168(77.1)$ & 534 (81.7) & \\
\hline$\geq 4000$ & $50(22.9)$ & $123(15.7)$ & & $50(22.9)$ & $120(11.3)$ & \\
\hline Smoking & & & 0.722 & & & 0.445 \\
\hline No & $156(71.6)$ & $552(70.3)$ & & $156(71.6)$ & $450(68.8)$ & \\
\hline Yes & $62(28.4)$ & 233 (29.7) & & $62(28.4)$ & $204(31.2)$ & \\
\hline Treatment & & & 0.568 & & & 0.334 \\
\hline RT & $90(55.5)$ & $336(42.8)$ & & $90(41.3)$ & $288(44.0)$ & \\
\hline CCRT & $78(45.5)$ & $300(38.2)$ & & $78(35.8)$ & $251(38.4)$ & \\
\hline $\mathrm{ICT}+\mathrm{RT}$ & $19(8.7)$ & $63(8.0)$ & & $19(8.7)$ & $49(7.5)$ & \\
\hline $\mathrm{ICT}+\mathrm{CCRT}$ & $31(14.2)$ & $86(11.0)$ & & $31(14.2)$ & $66(10.1)$ & \\
\hline
\end{tabular}

$p$ value $<0.05$ indicates a statistically significant difference

Abbreviations: $y r$, year; $E B V$, Epstein-Barr virus; $R T$, radiotherapy; $C C R T$, concurrent chemoradiotherapy; $I C T$, induction chemotherapy

(3.4\%) patients' deaths resulted from NPC progression and $4(0.6 \%)$ patients died from other reasons. In addition, $60(9.2 \%)$ patients developed disease progression, including $23(3.5 \%)$ cases of local occurrence, 17 (2.6\%) cases of regional occurrence, and $30(4.6 \%)$ cases of distant metastases.

The 5-year OS rate in the PET/CT group was $97.9 \%$ (95\% CI 96.9-98.9) in comparison with 96.4\% (95\% CI 95.6-97.2) in the CWU group ( $p=0.170$ ) (Fig. 4a). The 5-year LRFS and RRFS rates were $98.9 \%$ and $98.4 \%$ in the PET/CT cohort, and $98.2 \%$ and $97.8 \%$ in the CWU cohort ( $p=0.928$ and 0.409 , respectively) (Fig. 5). Similarly, the survival outcomes showed no significant difference between the PET/CT and CWU groups in terms of PFS and DMFS (5-year PFS: $93.8 \%$ vs. 91.7\%, $p=$
0.288; 5-year DMFS: 97.7\%vs. 95.3\%, $p=0.267$ ) (Fig. $4 \mathrm{~b}, \mathrm{c})$.

\section{Multivariate analysis}

In multivariate analysis, age, gender, $\mathrm{T}$ category, $\mathrm{N}$ category, clinical stage, smoking, EBV DNA level, treatment modality, and PET/CT application were considered as covariates and entered into Cox's proportional hazards model $(n=872)$. As shown in Table 3 , the use of PET/CT was not associated with improved OS $(p=0.262), \operatorname{PFS}(p=$ $0.196)$, LRRFS $(p=0.526)$, and DMFS $(p=0.182)$ in early-stage NPC. Age $(\mathrm{HR}=3.16,95 \%$ CI 1.44-6.92, $p=0.004)$ and $\mathrm{N}$ category $(\mathrm{HR}=4.14,95 \% \mathrm{CI} 1.67-$ $10.16, p=0.002$ ) were evaluated as independent 
Fig. 4 Kaplan-Meier survival curves for 872 stage I-II NPC patients stratified by the implementation of PET/CT: (a) overall survival, (b) progression-free survival, (c) distant metastasis-free survival a

$$
+\mathrm{CWU} \perp \mathrm{PETCT}+\mathrm{CWU}
$$
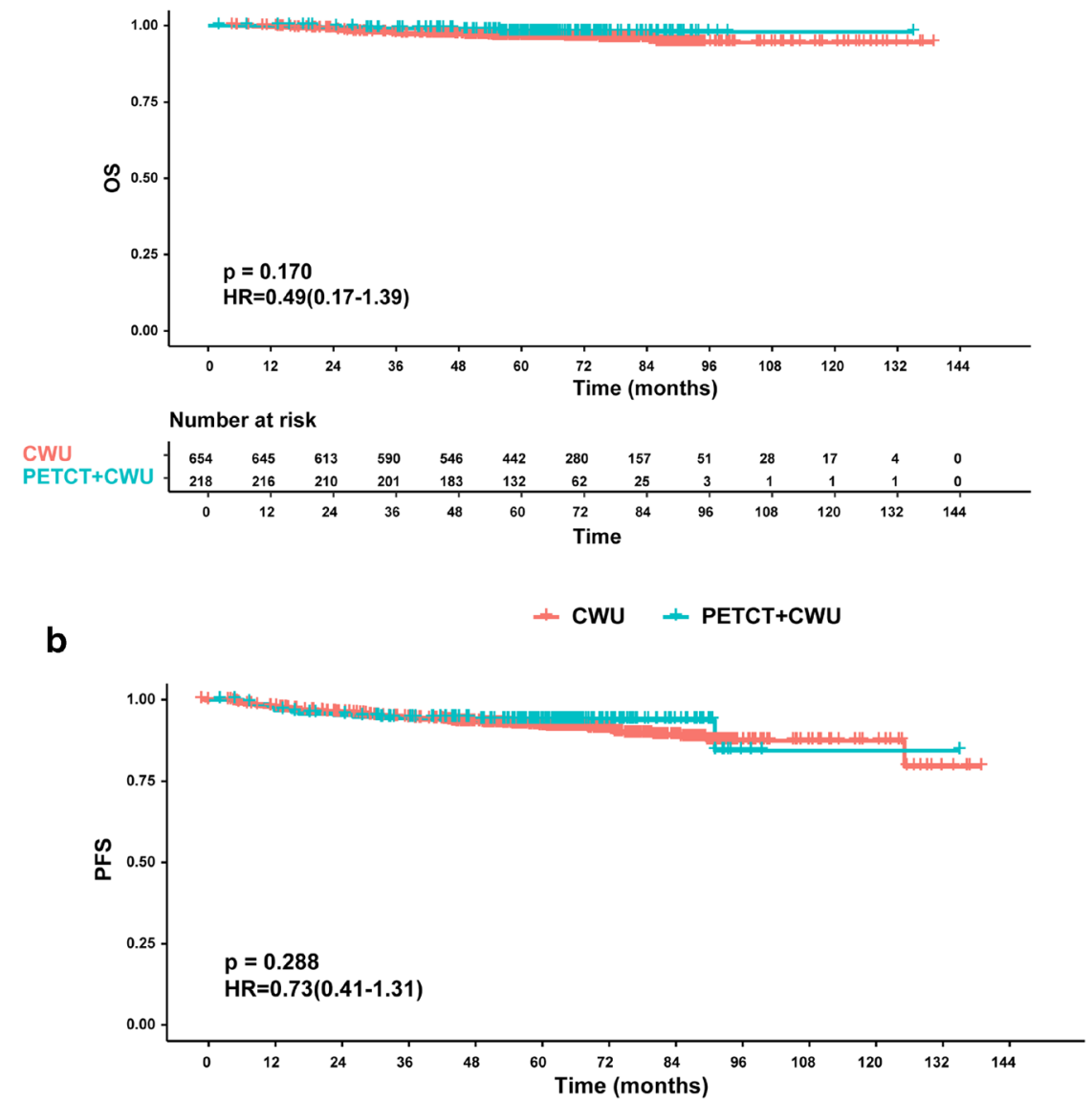

Number at risk

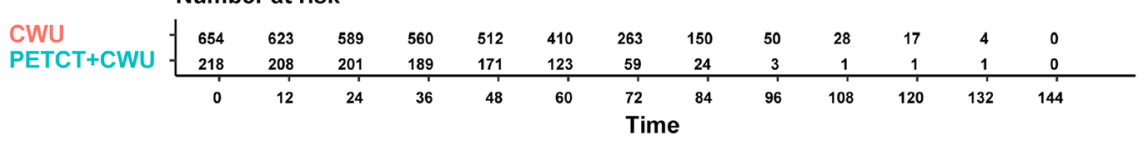

$+\mathrm{CWU}+\mathrm{PETCT}+\mathrm{CWU}$

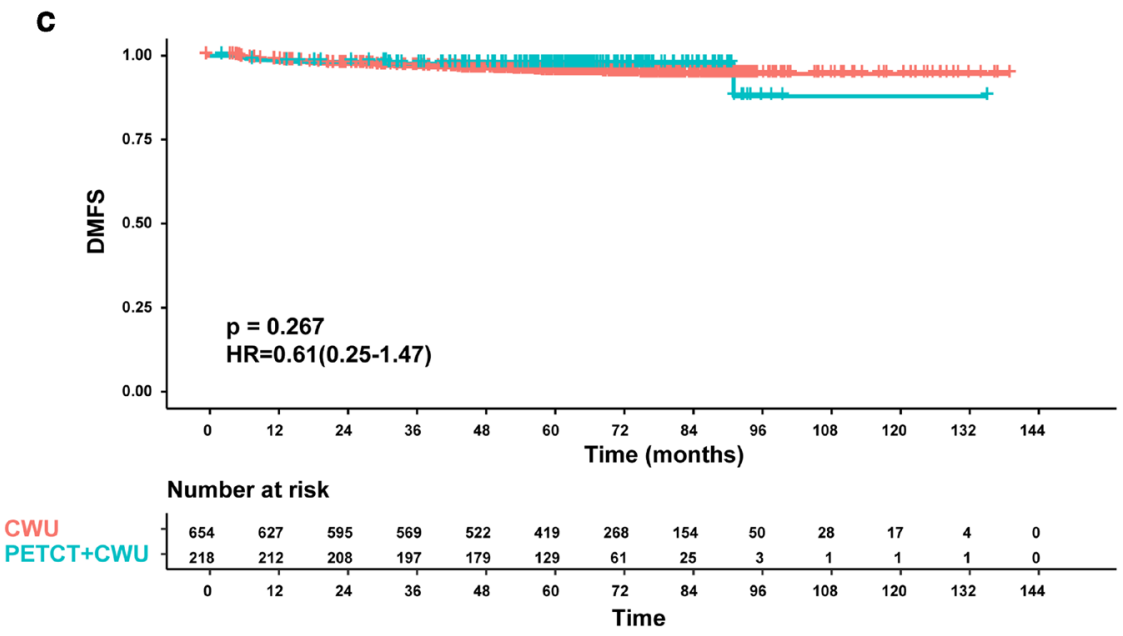

prognostic factors for OS. Two covariates were identified as independent prognostic factors for PFS, namely, N category $(\mathrm{HR}=2.44,95 \% \mathrm{CI} 1.48-4.00, p<0.001)$ and EBV DNA level $(\mathrm{HR}=1.74,95 \%$ CI $1.01-2.97, p=$
0.042). $\mathrm{N}$ category $(\mathrm{HR}=3.50,95 \%$ CI $1.73-7.07$, $p<0.001)$ and EBV DNA level $(\mathrm{HR}=3.55,95 \% \mathrm{CI}$ $1.84-6.85, p<0.001$ ) were found to be independent prognostic factors for LRRFS and DMFS, respectively. 
a

$\pm \mathrm{CWU} \perp \mathrm{PETCT}+\mathrm{CWU}$

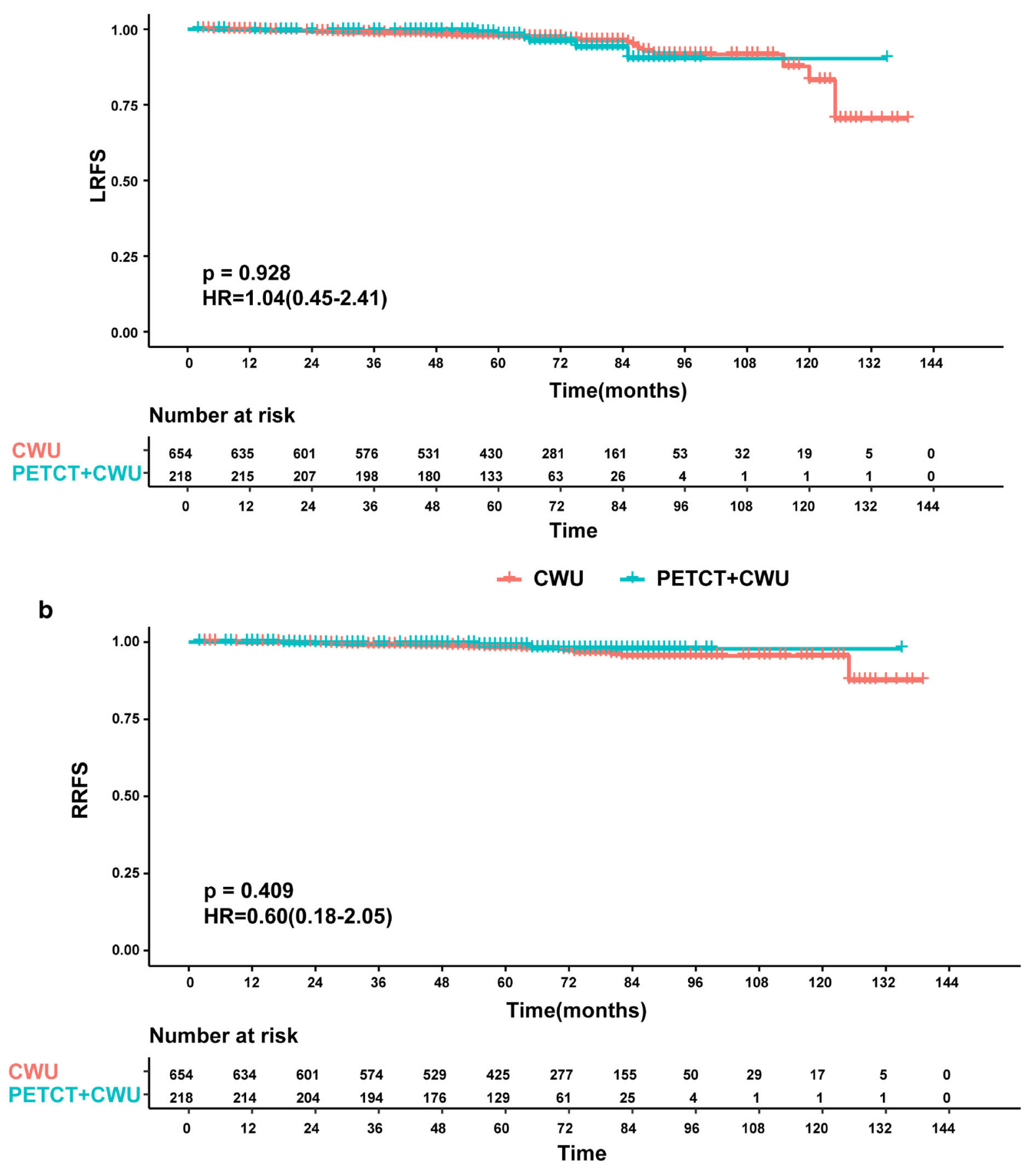

Fig. 5 Kaplan-Meier survival curves for 872 stage I-II NPC patients stratified by the implementation of PET/CT: (a) local relapse-free survival, (b) regional relapse-free survival

\section{Discussion}

Although many studies have clarified the significant value of $\mathrm{PET} / \mathrm{CT}$ in the initial imaging of patients with locoregional advanced NPC $[3,18]$, this technique may not play an equally significant role for stage I-II NPC in actual clinical practice. This is a nest case-control study to evaluate the clinical benefit of PET/CT in early-stage NPC over initial imaging with a long-term follow-up time. Consistent with previous studies $[6,19-21]$, we verified that PET/CT was superior to MRI in detecting CLN metastases but inferior in diagnosing RLN involvement. Nevertheless, we also found that the survival outcomes of NPC patients in survival analysis did not differ depending on whether PET/CT was performed. 
Table 3 Multivariable Cox models for PSM dataset $(n=872)$

\begin{tabular}{|c|c|c|c|c|c|c|c|c|}
\hline & \multicolumn{2}{|l|}{ PFS } & \multicolumn{2}{|l|}{ OS } & \multicolumn{2}{|l|}{ LRRFS } & \multicolumn{2}{|l|}{ DMFS } \\
\hline & $\begin{array}{l}\text { HR } \\
(95 \% \mathrm{CI})\end{array}$ & $p$ value & $\begin{array}{l}\mathrm{HR} \\
(95 \% \mathrm{CI})\end{array}$ & $p$ value & $\begin{array}{l}\text { HR } \\
(95 \% \mathrm{CI})\end{array}$ & $p$ value & $\begin{array}{l}\mathrm{HR} \\
(95 \% \mathrm{CI})\end{array}$ & $\begin{array}{l}p \\
\text { value }\end{array}$ \\
\hline Age & & NS & & 0.004 & & NS & & NS \\
\hline$\leq 45$ & & & Reference & & & & & \\
\hline$>45$ & & & $3.16(1.44-6.92)$ & & & & & \\
\hline $\mathrm{N}$ stage & & $<0.001$ & & 0.002 & & $<0.001$ & & NS \\
\hline N0 & Reference & & Reference & & Reference & & & \\
\hline $\mathrm{N} 1$ & $2.44(1.48-4.00)$ & & $4.14(1.67-10.16)$ & & $3.50(1.73-7.07)$ & & & \\
\hline EBV DNA & & 0.042 & & NS & & & & $<0.001$ \\
\hline$<4000$ & Reference & & & & & & Reference & \\
\hline$\geq 4000$ & $1.74(1.01-2.97)$ & & & & & & $3.55(1.84-6.85)$ & \\
\hline PET/CT obtained & & 0.262 & & 0.196 & & 0.526 & & 0.182 \\
\hline Yes & Reference & & Reference & & Reference & & Reference & \\
\hline No & $0.72(0.40-1.28)$ & & $0.56(0.23-1.35)$ & & $0.79(0.38-1.64)$ & & $0.49(0.17-1.40)$ & \\
\hline
\end{tabular}

$p$ value $<0.05$ indicates a statistically significant difference

Abbreviations: PFS, progression-free survival; OS, overall survival; LRRFS, locoregional relapse-free survival; DMFS, distant metastasis-free survival

In the present study, significant discrepancies existed in PET/CT and MRI in assessing the involvement of parapharyngeal space and the RLNs and CLNs. On the basis of MRI diagnosis, PET/CT altered $\mathrm{T}$ staging in $36.2 \%$ (79) patients, with $4.1 \%$ (9) being upstaged and $32.1 \%$ (70) being downstaged. Our results implied that $\mathrm{PET} / \mathrm{CT}$ was not as sensitive as MRI in detecting parapharyngeal space invasion, which was concordant with some previous studies [3]. This might be an outcome of the spillover effect of PET/CT and low FDG uptake in early-stage NPC. In terms of 9 patients with upstaged $\mathrm{T}$ category by PET/CT imaging but did not treat based on it, no patients developed local recurrence until the last follow-up. This might further imply the false-positive results of PET/CT. In this study, PET/CT staging did not change patients' major treatment strategy. We assumed that the use of PET/CT might change some patients' chemotherapy regimens but this is a retrospective study so that we could not be able to obtain the exact percentage. However, according to previous studies and our data, PET/CT is inferior to MRI in detecting soft tissue lesions. Moreover, the small portion changes of treatment regimens did not result in patients' survival difference based on our long-term follow-up. Therefore, we concluded that PET/CT staging did not change early-stage patients' major treatment strategy.

The fact that MRI is superior to PET/CT for detecting metastatic RLNs has been verified by a previous study [22]; the same conclusion was drawn in the current study. Among the 79 inconsistent results obtained from PET/CT and MRI, MRI yielded correct diagnoses in 72 patients. Besides, we found that PET/
CT was inclined to miss malignant RLNs because they were nearly connected with an adjacent primary tumor in many cases.

CLN metastasis plays a critical role in NPC due to its high incidence rate and independent prognostic value. It is also of vital importance in staging and prognostic classification of early-stage NPC [23, 24]. For the cases showing inconsistent lymph node results between PET/CT and MRI, we found that $\mathrm{PET} / \mathrm{CT}$ was more accurate than MRI in diagnosing CLN lesion. Although the functional imaging ability of PET/CT facilitates detection of CLN metastasis, it simultaneously caused difficulties in discriminating metastatic CLNs from inflammatory lymph nodes.

Distant metastasis is one of the most crucial factors guiding treatment planning in oncology which necessitates the accurate diagnosis [25]. Yen [13] demonstrated that the $\mathrm{N}$ category of NPC strongly influenced the possibility of distant metastasis. In our study, CWU and PET/CT did not detect any abnormality of primary distant metastasis at initial imaging, and only one patient developed liver metastasis within 1 year. Considering the low incidence rate of distant metastasis among parents with early-stage NPC, we inferred that PET/ CT may not offer benefits over CWU for detecting distant metastasis in early-stage NPC. Although we found that PET/ CT was superior to MRI in detecting CLN metastases, no association was observed between survival benefit and the application of pre-treatment PET/CT. It might be explained by the fact that PET/CT's use did not change patients' main treatment courses and all patients received prophylactic cervical irradiation. Changing the radiotherapy strategies due to $\mathrm{N}$ category might affect the quality of life; however, we could not be able to make an analysis. Because this is a retrospective 
study, we could not be able to compare patients' quality of life brought by use of PET/CT with patients who did not.

Our study had several limitations. Firstly, the SOT for lymph node metastasis in this study was defined by the change of size according to MRI follow-up instead of pathology which was regarded as the gold standard for diagnosis. Secondly, the lesion of parapharyngeal space that lacked SOT was due to the difficulty in obtaining histopathology result. Thirdly, we could not be able to obtain the effect of radiotherapy strategies on patients' life quality because the retrospective study and prospective study need to be carried out. Finally, all patients were from a single center in an epidemic area which prompted further verification from other institutions.

In conclusion, this study shows that PET/CT has little value in initial imaging for early-stage NPC patients. Therefore, this expensive imaging test should be used pragmatically, and its usage should be based on well-designed prospective studies targeting specific indications. Cost-effectiveness analyses of PET/CT scans according to clinical indications are also warranted.

Supplementary Information The online version contains supplementary material available at https://doi.org/10.1007/s00330-020-07478-1.

Funding This work was supported by grants from the National Key R\&D Program of China (2017YFC0908500, 2017YFC1309003), the National Natural Science Foundation of China (No. 81425018, No. 81672868, No. 81602371), the Sci-Tech Project Foundation of Guangzhou City (201707020039), the Sun Yat-sen University Clinical Research 5010 Program, the Special Support Plan of Guangdong Province (No. 2014TX01R145), the Natural Science Foundation of Guangdong Province (No. 2017A030312003, No.2017A030312003), the Sci-Tech Project Foundation of Guangdong Province (No. 2014A020212103), the Health \& Medical Collaborative Innovation Project of Guangzhou City (No. 201400000001, No.201803040003), the Planned Science and Technology Project of Guangdong Province (2019B020230002), the National Science \& Technology Pillar Program during the Twelfth Five-year Plan Period (No. 2014BAI09B10), the PhD Start-up Fund of Natural Science Foundation of Guangdong Province (2016A030310221), the cultivation foundation for the junior teachers in Sun Yat-sen University(16ykpy28), and the Fundamental Research Funds for the Central Universities.

\section{Compliance with ethical standards}

Guarantor The scientific guarantor of this publication is Lin-Quan Tang

Conflict of interest The authors of this manuscript declare no relationships with any companies whose products or services may be related to the subject matter of the article.

Statistics and biometry One of the authors has significant statistical expertise.

Informed consent Written informed consent was obtained from all subjects (patients) in this study.

Ethical approval Institutional Review Board approval was obtained.
Methodology

- retrospective

- observational

- performed at one institution

Open Access This article is licensed under a Creative Commons Attribution 4.0 International License, which permits use, sharing, adaptation, distribution and reproduction in any medium or format, as long as you give appropriate credit to the original author(s) and the source, provide a link to the Creative Commons licence, and indicate if changes were made. The images or other third party material in this article are included in the article's Creative Commons licence, unless indicated otherwise in a credit line to the material. If material is not included in the article's Creative Commons licence and your intended use is not permitted by statutory regulation or exceeds the permitted use, you will need to obtain permission directly from the copyright holder. To view a copy of this licence, visit http://creativecommons.org/licenses/by/4.0/.

\section{References}

1. Wee JT, Ha TC, Loong SL, Qian CN (2010) Is nasopharyngeal cancer really a "Cantonese cancer"? Chin J Cancer 29(5):517-526

2. Cao SM, Simons MJ, Qian CN (2011) The prevalence and prevention of nasopharyngeal carcinoma in China. Chin J Cancer 30(2): 114-119

3. Ng SH, Chan SC, Yen TC et al (2009) Staging of untreated nasopharyngeal carcinoma with PET/CT: comparison with conventional imaging work-up. Eur J Nucl Med Mol Imaging 36(1):12-22

4. Liu FY, Lin CY, Chang JT et al (2007) 18F-FDG PET can replace conventional work-up in primary $\mathrm{M}$ staging of nonkeratinizing nasopharyngeal carcinoma. J Nucl Med 48(10):1614-1619

5. Yen TC, Chang JT, Ng SH et al (2005) The value of 18F-FDG PET in the detection of stage M0 carcinoma of the nasopharynx. J Nucl Med 46(3):405-410

6. Chang JT, Chan SC, Yen TC et al (2005) Nasopharyngeal carcinoma staging by (18)F-fluorodeoxyglucose positron emission tomography. Int J Radiat Oncol Biol Phys 62(2):501-507

7. Cheuk DK, Sabin ND, Hossain M et al (2012) PET/CT for staging and follow-up of pediatric nasopharyngeal carcinoma. Eur J Nucl Med Mol Imaging 39(7):1097-1106

8. Tang LQ, Chen QY, Fan W et al (2013) Prospective study of tailoring whole-body dual-modality [18F]fluorodeoxyglucose positron emission tomography/computed tomography with plasma Epstein-Barr virus DNA for detecting distant metastasis in endemic nasopharyngeal carcinoma at initial staging. J Clin Oncol 31(23): 2861-2869

9. Li Z, Li Y, Li N, Shen L (2019) Positron emission tomography/ computed tomography outperforms MRI in the diagnosis of local recurrence and residue of nasopharyngeal carcinoma: an update evidence from 44 studies. Cancer Med 8(1):67-79

10. National Comprehensive Cancer Network. Head and neck cancers (Version 3.2019). https://www.nccn.org/professionals/physician gls/pdf/head-and-neck.pdf. Accessed 16 Sept 2019

11. King AD, Ahuja AT, Leung SF et al (2000) Neck node metastases from nasopharyngeal carcinoma: MR imaging of patterns of disease. Head Neck 22(3):275-281

12. Zhou X, Ou X, Yang Y et al (2018) Quantitative metastatic lymph node regions on magnetic resonance imaging are superior to AJCC $\mathrm{N}$ classification for the prognosis of nasopharyngeal carcinoma. $\mathrm{J}$ Oncol 2018:9172585

13. Liu FY, Chang JT, Wang HM et al ( $\left.\begin{array}{llll}2 & 0 & 0 & 6\end{array}\right)$ $[18 \mathrm{~F}]$ Fluorodeoxyglucose positron emission tomography is more sensitive than skeletal scintigraphy for detecting bone metastasis 
in endemic nasopharyngeal carcinoma at initial staging. J Clin Oncol 24(4):599-604

14. Koshy M, Paulino AC, Howell R, Schuster D, Halkar R, Davis LW (2005) F-18 FDG PET-CT fusion in radiotherapy treatment planning for head and neck cancer. Head Neck 27(6):494-502

15. Eisenhauer EA, Therasse P, Bogaerts J et al (2009) New response evaluation criteria in solid tumours: revised RECIST guideline (version 1.1). Eur J Cancer 45(2):228-247

16. Zhang GY, Liu LZ, Wei WH, Deng YM, Li YZ, Liu XW (2010) Radiologic criteria of retropharyngeal lymph node metastasis in nasopharyngeal carcinoma treated with radiation therapy. Radiology. 255(2):605-612

17. Zhang Y, Chen L, Hu G et al (2019) Gemcitabine and cisplatin induction chemotherapy in nasopharyngeal carcinoma. N Engl J Med 381(12):1124-1135

18. King AD, Ma BB, Yau YY et al (2008) The impact of 18F-FDG $\mathrm{PET} / \mathrm{CT}$ on assessment of nasopharyngeal carcinoma at diagnosis. Br J Radiol 81(964):291-298

19. Comoretto M, Balestreri L, Borsatti E, Cimitan M, Franchin G, Lise $M$ (2008) Detection and restaging of residual and/or recurrent nasopharyngeal carcinoma after chemotherapy and radiation therapy: comparison of MR imaging and FDG PET/CT. Radiology 249(1): 203-211

20. Adams S, Baum RP, Stuckensen T, Bitter K, Hor G (1998) Prospective comparison of $18 \mathrm{~F}-\mathrm{FDG}$ PET with conventional imaging modalities (CT, MRI, US) in lymph node staging of head and neck cancer. Eur J Nucl Med 25(9):1255-1260

21. Braams JW, Pruim J, Freling NJ et al (1995) Detection of lymph node metastases of squamous-cell cancer of the head and neck with FDG-PET and MRI. J Nucl Med 36(2):211-216

22. Tang LL, Ma J, Chen Y et al (2007) The values of MRI, CT, and PET-CT in detecting retropharyngeal lymph node metastasis of nasopharyngeal carcinoma. Ai Zheng 26(7):737-741

23. Zhang LL, Zhou GQ, Li YY et al (2017) Combined prognostic value of pretreatment anemia and cervical node necrosis in patients with nasopharyngeal carcinoma receiving intensity-modulated radiotherapy: a large-scale retrospective study. Cancer Med 6(12): 2822-2831

24. Du XJ, Tang LL, Mao YP et al (2016) Circulating EBV DNA, globulin and nodal size predict distant metastasis after intensitymodulated radiotherapy in stage II nasopharyngeal carcinoma. J Cancer 7(6):664-670

25. Sonnenschein C, Soto AM (2015) Cancer metastases: so close and so far. J Natl Cancer Inst 107(11):26283653. https://doi.org/10. 1093/jnci/djv236

Publisher's note Springer Nature remains neutral with regard to jurisdictional claims in published maps and institutional affiliations. 